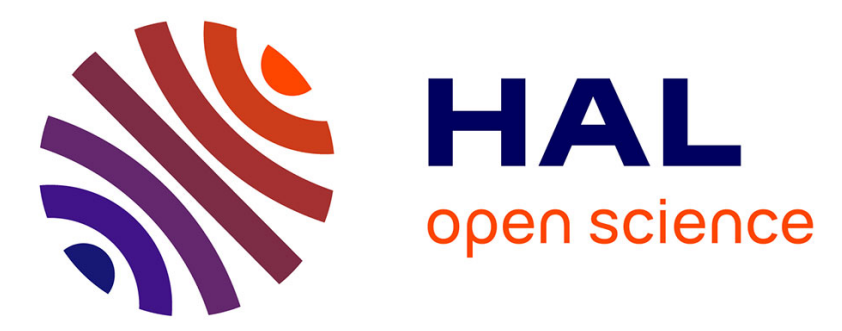

\title{
Time-varying autoregressive spectral estimation for ultrasound attenuation in tissue characterization
}

Jean-Marc Girault, Frédéric Ossant, Abdeldjalil Ouahabi, Denis Kouamé, Frédéric Patat

\section{- To cite this version:}

Jean-Marc Girault, Frédéric Ossant, Abdeldjalil Ouahabi, Denis Kouamé, Frédéric Patat. Timevarying autoregressive spectral estimation for ultrasound attenuation in tissue characterization. IEEE Transactions on Ultrasonics, Ferroelectrics and Frequency Control, 1998, 45 (3), pp.650-659. 10.1109/58.677609 . hal-01076705

\section{HAL Id: hal-01076705 https://hal.science/hal-01076705}

Submitted on 2 Mar 2016

HAL is a multi-disciplinary open access archive for the deposit and dissemination of scientific research documents, whether they are published or not. The documents may come from teaching and research institutions in France or abroad, or from public or private research centers.
L'archive ouverte pluridisciplinaire HAL, est destinée au dépôt et à la diffusion de documents scientifiques de niveau recherche, publiés ou non, émanant des établissements d'enseignement et de recherche français ou étrangers, des laboratoires publics ou privés. 


\title{
Time-Varying Autoregressive Spectral Estimation for Ultrasound Attenuation in Tissue Characterization
}

\author{
Jean-Marc Girault, Frédéric Ossant, Abdeljalil Ouahabi, Denis Kouamé, and Frédéric Patat \\ LUSSI, GIP Ultrasons, Tours, France (e-mail: imgirault@univ-tours.fr) \\ DOI : $10.1109 / 58.677609$
}

\begin{abstract}
In the eld of biological tissue characteri-zation, fundamental acoustic attenuation properties have been demonstrated to have diagnostic importance. Attenu-ation caused by scattering and absorption shifts the instan-taneous spectrum to the lower frequencies. Due to the time-dependence of the spectrum, the attenuation phenomenon is a time-variant process. This downward shift may be eval-uated either by the maximum energy frequency of the spec-trum or by the center frequency.

In order to improve, in strongly attenuating media, the results given by the short-time Fourier analysis and the short-time parametric analysis, we propose two approaches adapted to this time-variant process: an adaptive method and a time-varying method. Signals backscattered by an homogeneous medium of scatterers are modeled by a computer algorithm with attenuation values ranging from 1 to 5 $\mathrm{dB} / \mathrm{cmMHz}$ and a $45 \mathrm{MHz}$ transducer center frequency.

Under these conditions, the preliminary results obtained with the proposed time-variant methods, compared with the classical short-time Fourier analysis and the short-time auto-regressive (AR) analysis, are superior in terms of stan-dard deviation (SD) of the attenuation coefficient estimate. This study, based on nonstationary AR spectral estimation, promises encouraging perspectives for in vitro and in vivo applications both in weakly and highly attenuating media.
\end{abstract}

\section{Introduction}

Great progress has been made recently in high frequency ultrasound imaging [1]. In particular, several studies have shown the interest of echographic exploration for frequencies ranging from 20 to $100 \mathrm{MHz}$ in dermatol-ogy and ophthalmology [2]. These results are extending the eld of application of tissue characterization.

As an acoustic pulse propagates through soft tissue, it experiences an attenuation-dependent frequency-shift.

Several techniques can be used to determine the frequency shift of backscattered signals. Fourier analysis and parametric spectral estimation [3], [4], commonly em-ployed in the tissue characterization field, give similar re-sults in weakly attenuating media. These two methods are based upon local stationary signal assumptions and use a sliding window technique. In the reflection mode and only in highly attenuating media, it has been shown [5] that the parametric spectral analysis, and more precisely the autoregressive spectral analysis, provides a better estima-tion of attenuation than the Fourier approach in terms of relative error. This parametric analysis consists in model-ing the echographic signal as the output of a linear Iter driven by a white Gaussian noise. This Iter, referred to as $A R$, is a linear combination of the previous samples (Regressive) of the output itself (Auto).

To improve the performances obtained by the conventional parametric approach and the Fourier approach, both in moderately and highly attenuating media, another class of parametric spectral analysis is investigated: the nonstationary autoregressive spectral analysis.

In this paper, we propose two new approaches of para-metric spectral analysis for attenuation estimation: an adaptive method and a time-varying recursive method. The first method computes and updates the AR parame-ters at each sampling time using the prediction error (the di erence between the echographic signal and the esti-mated output of the AR process) and a constant forgetting factor. This forgetting factor is equivalent to introducing an exponential window which forgets the previous sam-ples. In the second one, each AR parameter is allowed to change in time by assuming it is a linear combination of a known set of time functions.

The fundamental di erence between the algorithm structures of nonstationary and conventional (AR or Fourier) analyses is that the proposed algorithms directly give $N$ spectra when $N$ signal samples are processed. In the classical analyses, the signal composed of $N$ samples is truncated into $P$ windows (for example, 16 windows, with $50 \%$ overlapping) and $P$ spectra are computed $(P<N)$. The two new methods applied to backscattered simulated signals are compared to the short time Fourier analysis and the short time AR analysis, for di erent attenuation coefficient values ( 1 to $5 \mathrm{~dB} / \mathrm{cmMHz}$ ) with a $45 \mathrm{MHz}$ ultrasonic transducer center frequency.

This paper is organized as follows: in Section II, we describe how the backscattered signals are modeled, and we introduce the attenuation estimation. We review the AR modeling and the parameters computation in Sections III and IV. Section V is concerned with the spectral estimation. Results and interpretation are performed in Section VI, and Section VII is the conclusion.

\section{Backscattered Signal Modeling and}

\section{Attenuation Estimation}

\section{A. Backscattered Signal Modeling}

A reasonable accurate model for signals backscattered by a biological tissue [6] consists of a linear transformation of components representing di erent contributions: on the one hand, the measuring system impulse response (basic ultrasonic wavelet), and on the other hand, the scattering and the absorption function of the explored medium. Consequently, the reflected signal $x(t)$ from a given region of a medium can be represented as a function of the ultrasound wavelet $e(t)$ and a time-varying impulse response $h(t ;)$ of the explored tissue:

$$
X(f)=E(f) \sum_{i=1}^{L} \xi_{i} e^{-\frac{\alpha(f) c \tau_{i}}{8.68}} e^{-j 2 \pi f \tau_{i}}
$$

where $G$ denotes a linear function.

An example of backscattered signal, as well as an over-simpli ed scheme of our simulated medium, is given in Fig. 1.

We assume that scatterers have a random spatial distribution and are point scatterers. This assumption implies that the shape of scatterers is not taken into account. We also consider that attenuation is homogeneous and that only simple scattering takes place (that there is no multi-ple scattering, i.e., the Born's approximation is satis ed).

Concerning the transducer, we assume that the power spectral density of the emitted signal $e(t)$ has a Gaussian form (see Fig. 2); this implies that its spectral variance $s^{2}$ is constant versus depth. Moreover, we neglect the diffraction effects. 


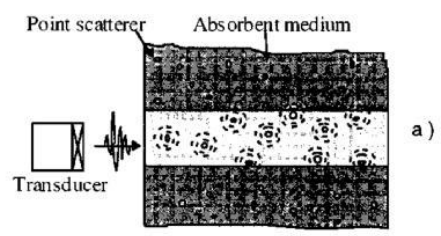

Typically backscattered signal

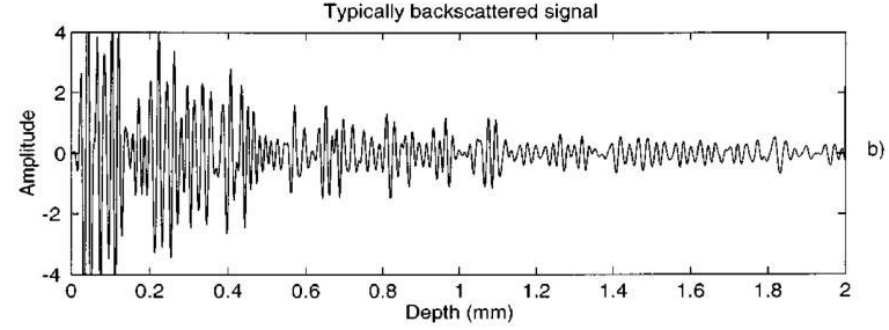

Fig. 1. The medium (a), typically backscattered signal (b).

Accordingly, the spectrum of the backscattered signal by $L$ scatterers is given by:

$X(f)=E(f) \sum_{i=1}^{L} \xi_{i} e^{-\frac{\alpha(f) c \tau_{i}}{8.68}} e^{-j 2 \pi f \tau_{i}}$

where is the ultrasonic attenuation, $E(f)$ is the transfer function of the transducer, and $i$ is a random amplitude uniform in the interval $(0 ; 1)$.

The summation term represents the composite nature of the backscattered echoes detected at the random arrival times $\tau_{i}$, where $\tau_{i}$ and $\xi_{i}$ are assumed to be uncorrelated random variables.

The performances of the di erent proposed methods are tested on uncorrelated simulated A mode echo lines. Di erent media with attenuations ranging from 1 to 5 $\mathrm{dB} / \mathrm{cmMHz}$ are considered. The sampling frequency $f_{S}$ is $400 \mathrm{MHz}$, the transducer center frequency $f_{0}$ is $45 \mathrm{MHz}$ and the spectral standard deviation $\sigma_{S}$ is $7.46 \mathrm{MHz}$. Each simulation contains 256 A-lines of 1024 samples, with a speed of ultrasound of $1530 \mathrm{~m} / \mathrm{s}$ leading to a depth of ex-plored tissue around $2 \mathrm{~mm}$.

\section{B. Attenuation Estimation}

Suppose that the attenuation $(\mathrm{dB} / \mathrm{cm})$ is linearly dependent on frequency [5], [7]. We can de ne the slope of attenuation or the attenuation coefficient $(\mathrm{dB} / \mathrm{cmMHz})$ :

$$
\alpha=\beta f \text {. }
$$

Consequently, the power spectral density (PSD) of $x(t)$ can be expressed as a function of frequency and depth $d$ :

$$
S_{x x}(f, d)=S_{e}(f) e^{-4 \beta f d}
$$
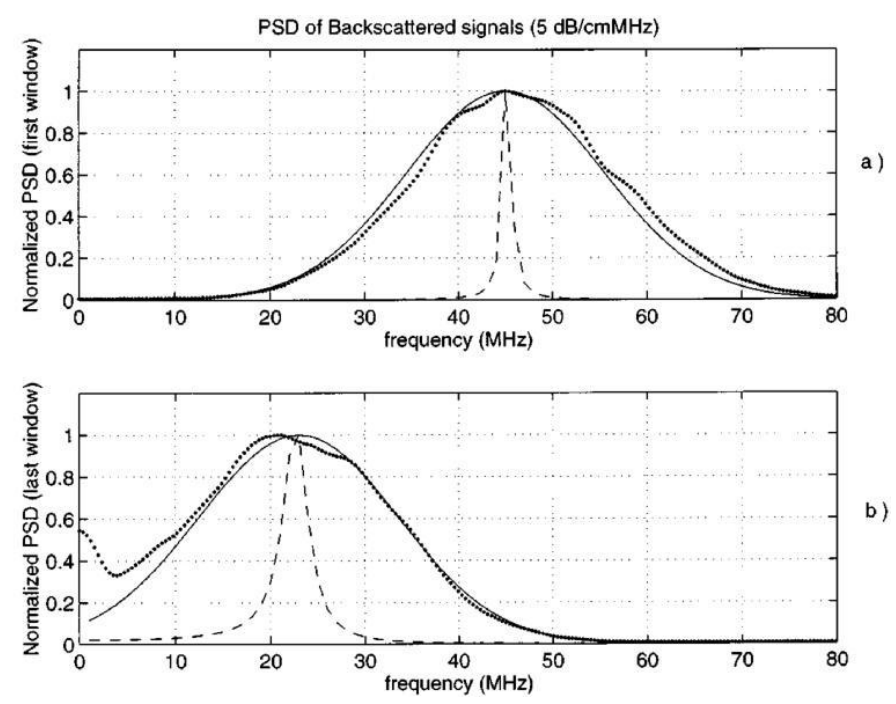

Fig. 2. Evolution of the normalized PSD of the reflected signal in the rst analyzing window (a) and in the last analyzing window (b). Theoretical PSD (solid line), averaged PSD evaluated by the FFT (dotted line) and second-order AR PSD (dashed line). The simulation conditions are: $f_{0}=45 \mathrm{MHz}, s=7: 46 \mathrm{MHz},=5 \mathrm{~dB} / \mathrm{cmMHz}$ and $f_{S}=$ $400 \mathrm{MHz}$.

where $S_{e}(f)$ is the PSD of $e(t)$.

The frequency-shift, due to the attenuation e ects, appears in all depth-varying frequencies and particularly for the maximum energy frequency (the frequency corresponding to the maximum of the PSD) and the center frequency (de ned as the centroid of the PSD). Because the PSD of the emitted signal $e(t)$ has a Gaussian form [8], the power spectrum of the reflected signal is given by:

$$
S_{x x}(f, d)=e^{-\frac{\left(f-f_{0}\right)^{2}}{2 \sigma_{s}^{2}}} e^{-4 \beta d f}
$$

where $f_{0}$ is the emitted transducer center frequency and $\sigma_{S}$ the spectral standard deviation that is related to the transducer bandwidth.

Now, let us focus our study on the maximum energy frequency derived by di erentiating (5) with respect to $f$ and setting it to zero:

$$
f_{\max }(d)=f_{0}-4 \beta d \sigma_{s}^{2}
$$

By differentiating (6) with respect to $t=2 d=c$ ( $c$ : speed of ultrasound) the slope of the attenuation is given by:

$$
\beta_{(\mathrm{dB} / \mathrm{cmMHz})}=\frac{-4.34}{c \sigma_{s}^{2}} \frac{d f_{\max }(t)}{d t}
$$

Expression (7) is identical to the one obtained in the case of the center frequency [5]. By using a linear regression, we can obtain the attenuation coefficient.

From (7), it is obvious that the better the estimation of the maximum energy frequency (or the centroid fre-quency), the better the attenuation coefficient estimation. In the most usual case and for small values of attenuation, the shorttime Fourier technique is used for this purpose. However, it is well-known that this technique has many limitations such as di culties to choose an analysis win-dow whose length takes into account nonstationarity in the signal, and lack of accuracy in frequency estimation. 

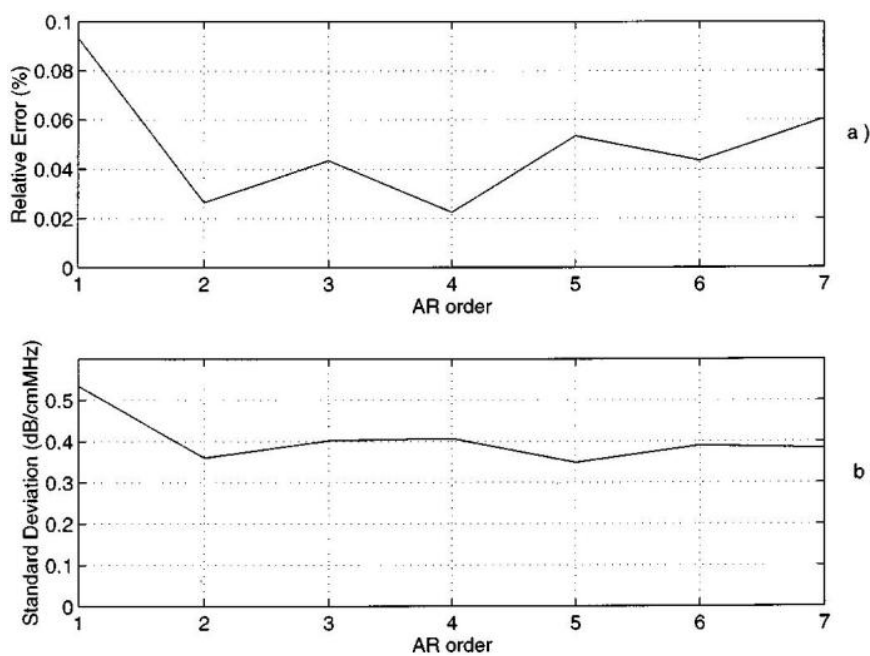

Fig. 3. Evolution of the relative error (a) and the standard devia-tion (b) of the attenuation coefficient estimate versus the AR order $p$. The simulation conditions are: $f_{0}=45 \mathrm{MHz}, s=7: 46 \mathrm{MHz},=5 \mathrm{~dB} / \mathrm{cmMHz}$, and $f_{S}=400 \mathrm{MHz}$ (same behavior for $=1,2,3,4 \mathrm{~dB} / \mathrm{cmMHz}$ ). The illustrated results are averaged with 256 radio-frequency lines.

Spectra computed with the FFT have a signi cant variance that contributes to increase the variance of the nal estimation of the attenuation coefficient.

To overcome these limitations, parametric analysis has been used in many elds and namely in speech processing, seismology, and Doppler ultrasound. Parametric tools have been recently applied to ultrasonic attenuation estimation [3], [5]. However, this approach requires a compromise between the accuracy that can only be achieved with large data segments and the time resolution required to track the spectrum, which can only be achieved with short data segments. Compared to FFT, it only reduces the relative error in highly attenuating media (no improvement in a weakly attenuating medium). In order to improve the standard deviation, we suggest the use of new methods that directly take into account the nonstationary signal character. These methods should give better results in cases such as the ones we encounter in a highly attenuating medium.

\section{Nonstationary Autoregressive Modeling}

The echographic signal $x(n)$, which is strongly nonsta tionary in highly attenuating media is digitized with a sampling frequency $f s$ in $N$ samples. This numerical signal is modeled as the output of a linear Iter driven by white Gaussian noise $u(n)$ with zero mean and variance $\sigma^{2}[9]$. It is given by:

$$
x(n)=-\sum_{i=1}^{p} a_{i}(n) x(n-i)+u(n)
$$

where $a_{i}(n)$ are the AR parameters at time $n$, where $n$ is related to distance by $d=n T_{s} c=2, c$ is the speed of ultrasound, $T_{S}$ is the sampling interval, and $p$ is the order of the AR model (or the number of parameters $a_{i}$ ). In practice, the order of the AR model must be chosen. For di er-ent values of the AR order $p$, we have computed the rel-ative error and the standard deviation of the attenuation coefficient estimate (see Fig. 3). In our study, it appears that a second-order AR process (AR2) is su cient. It is important to point out that the AR model matches the signal spectrum very closely near spectrum
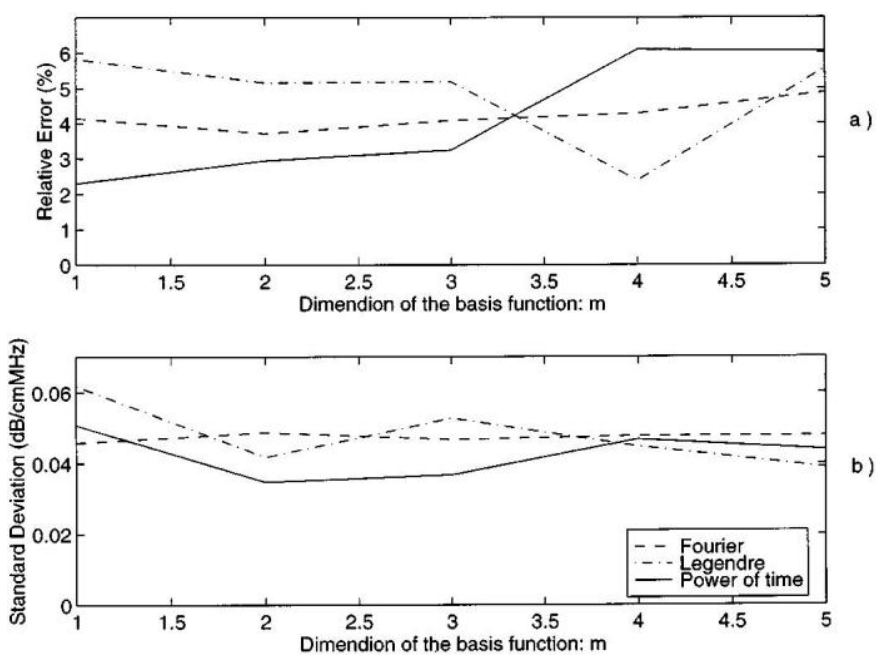

Fig. 4. Evolution of the relative error (a) and the standard devia-tion (b) of the attenuation coefficient estimate versus the dimension of the basis functions and for the three proposed basis functions.

Trigonometric (Fourier) basis function (dashed line), Legendre ba-sis function (dashdot line), and Power of time basis function (solid line).

The simulation conditions are: $f_{0}=45 \mathrm{MHz}, \sigma_{S}=7: 46 \mathrm{MHz}, \beta=5$

$\mathrm{dB} / \mathrm{cmMHz}$, and $f_{S}=400 \mathrm{MHz}$ (same behavior for $\beta=1,2,3,4$ $\mathrm{dB} / \mathrm{cmMHz}$ ). The illustrated results are averaged with 256 radiofrequency lines.

peaks, even if the order is low. Thus, a second-order AR model can e -ciently estimate the maximum energy frequency or center frequency of a signal.

If we suppose that the time-varying parameters are linear combinations of a set of deterministic basis timevarying functions $\mathrm{Fg}(\mathrm{n})$, then we transform a linear nonstationary problem into a linear stationary one by replacing a scalar process with a vector process [10]. In this case, time-varying AR parameters are expressed by:

$$
a_{i}(n)=\sum_{g=0}^{m} a_{i, g} F_{g}(n)
$$

where $a_{i ; g}$ are AR constant coefficients and $m$ is the dimension of time functions basis $F_{g}(n)$. The AR time-varying model for a nonstationary sample signal $x(n)$ is [10], [11]:

$$
x(n)=-\sum_{i=1}^{p} \sum_{g=0}^{m} a_{i, g} F_{g}(n-i) x(n-i)+u(n) .
$$

The number of unknowns is multiplied by $(m+1)$, but this seems a small price to pay compared to the bene $t$ of keeping the problem linear.

Several base functions (Appendix A) have been used in di erent elds, for example, in speech processing [10], [11]. Among all these bases, we decided to study the three most commonly used:

- Power of time functions [10] that usually are used in a quasi linear time-varying parameters evolution.

- Legendre functions [10] that are often used when pa-rameter evolution is rapid.

- Trigonometric functions [12] that are commonly used when time-varying parameters evolution is periodic.

In practice, the basis and its dimension must be chosen from the three bases above, we calculate for di erent val-es of the dimension $m$, the relative error (RE) and the standard 
deviation (SD) of the attenuation coefficient es-timate (Fig. 4). In this study, we found that the power of time functions with $m=2$ is the best choice in terms of both RE and SD as well as in terms of complexity. In-deed, the choice of superior dimensions only increases the algorithm complexity without signi cant improvement.

\section{AR Parameter Estimation}

There are many algorithms to compute AR parameters required for the spectrum estimation: Levinson, Burg, least-squares method, gradient method, Lattice Iter, Kalman Iter [9], [13], [14]. We will focus on the most popular one: the least-squares algorithm [14]. This method is applied with the simulation conditions presented in Sec-tion II.

\section{A. Batch Least-Squares Method with Sliding Window}

First, we recall the least-squares technique on which the following algorithms are based, and then the sliding window technique. Note that this technique is very close to the Burg's technique introduced by [5], [15].

Let us rewrite (8) in matrix form:

$$
x(n)=\phi_{n}^{T} \theta+u(n)
$$

and for $N$ data points:

$$
\begin{aligned}
& X=\phi^{T} \theta+U \\
& X^{T}=[x(n), \ldots, x(n-N)] \\
& \phi=\left[\phi_{n}^{T}, \ldots, \phi_{n-N}^{T}\right] \\
& \phi_{n}^{T}=[-x(n-1), \ldots,-x(n-p)] \\
& \theta=\left[a_{1}, \ldots, a_{p}\right]^{T} \\
& U=[u(n), \ldots, u(n-N)]^{T} \\
& \text { Estimation is given by: } \\
& \hat{X}=\phi^{T} \hat{\theta}
\end{aligned}
$$

where the hat indicates the estimated value.

The batch least-squares method evaluates the $a_{i}$ param-eters by minimizing the cost function:

$$
\begin{aligned}
& J=\left(X-\phi^{T} \hat{\theta}\right)^{T}\left(X-\phi^{T} \hat{\theta}\right) \\
& \left.\frac{\partial J}{\partial \hat{\theta}}\right|_{\theta=\hat{\theta}}=0 .
\end{aligned}
$$

The estimated parameters vector is:

$$
\hat{\theta}=\left(\phi^{T} \phi\right)^{-1} \phi^{T} X \text {. }
$$

Owing to the nonstationary character of the radio-frequency signal, a Hamming window is shifted (for ex-ample 16 windows, with $50 \%$ overlapping) with a constant step along the signal and in each window AR parameters are evaluated. Therefore, this method does not permit the determination of a new spectrum at each sample, but at each window. It implies a global estimation of the attenuation in a xed range of depths. Fig. 5 shows the evolution of the centroid frequency versus depth, both for the short time Fourier approach and for the short time AR approach. These curves were averaged for 256 radio-frequency lines.

\section{B. Recursive Weighted Least-Squares Method}

If long data sequences are available, it is possible to use a time-variant identi cation method. In this section, we de- scribe a time-variant algorithm that allows us to obtain a new set of parameters whenever a new sample is available. This is accomplished by updating the previously evaluated sample on the basis of the prediction error, and weighting by means of a forgetting factor. In this way, it is possi-ble to calculate an instantaneous spectrum, hence to study the spectral characteristics even under nonstationary conditions. In this case, the cost function becomes:

$$
J_{k}=\sum_{n=1}^{k} \lambda^{k-n}(x(n)-\hat{x}(n))^{2}
$$

where in practice $0: 95<\lambda<1$ and $k$ is the index of the last sample considered.

The general expression of the recursive algorithm is given by:

$$
\begin{aligned}
& {\left[\begin{array}{c}
\text { parameter } \\
\text { vector at } \\
\text { time } n
\end{array}\right]=\left[\begin{array}{c}
\text { parameter } \\
\text { vector at } \\
\text { time }(n-1)
\end{array}\right]+[\text { gain }]\left[\begin{array}{c}
\text { observation } \\
\text { vector }
\end{array}\right]\left[\begin{array}{c}
\text { prediction } \\
\text { error }
\end{array}\right]} \\
& \hat{\theta}_{n}=\hat{\theta}_{n-1}+P_{n} \quad \phi_{n} \quad \varepsilon_{n} .
\end{aligned}
$$

Due to the recursive nature of this algorithm, a transient region appears and it is required to initialize the gain $P_{n}$ and AR parameters. A standard initialization value of the gain is $P_{0}=10 /$ ( $/$ is an identity matrix) [13].

That is accomplished through the use of the forgetting factor in the expression of $P_{n}$ :

$$
\begin{aligned}
& P_{n}=\frac{P_{n-1}}{\lambda}\left(1-\frac{P_{n-1} \phi_{n} \phi_{n}^{T} P_{n-1}}{\lambda+\phi_{n}^{T} P_{n-1} \phi_{n}}\right) \\
& \varepsilon(n)=x(n)-\phi_{n}^{T} \hat{\theta}_{n-1} \\
& \hat{\theta}_{n}=\hat{\theta}_{n-1}+P_{n} \phi_{n} \varepsilon_{n} .
\end{aligned}
$$

Use of a constant forgetting factor is equivalent to the introducing of an exponential window. The length of this window indicates which of the past samples are e ectively taken into account. For example, if $=0.95$, there are 20 samples in the window whereas if $=0.99$ there are 100 samples. In this section, we have searched the forgetting factor that 0 ers the best trade-o between a good accuracy and a reduced statistical fluctuation on parameters estimation. In other words, for di erent attenuation coe cient values, we sought the value of that gives the lowest $\mathrm{RE}$ and SD of the attenuation coefficient estimate. Fig. 6 illustrates that the best forgetting factor is $=0.98$.

\section{Recursive Time-Varying Least-Squares Method}

Extension of the recursive weighted algorithm can be performed by multiplying the regression vector by the ba sis functions and increasing the AR order $p$ by $p(m+1)$ while setting the forgetting factor to one. By using (8), the algorithm becomes:

$$
\begin{aligned}
& x(n)=\varphi_{n-1}^{T} \hat{\theta}+u(n) \\
& \varphi_{n-1}^{T}=\phi_{n-1}^{T} V \\
& V=\left[F_{0, n-1}, \ldots, F_{m, n-p}\right]^{T} \\
& \hat{\theta}=\left[\hat{a}_{1,0} \ldots \hat{a}_{1, p} \ldots \hat{a}_{m, 0} \ldots \hat{a}_{m, p}\right]^{T} \\
& \text { where } \phi \text { is de ned in (14). }
\end{aligned}
$$




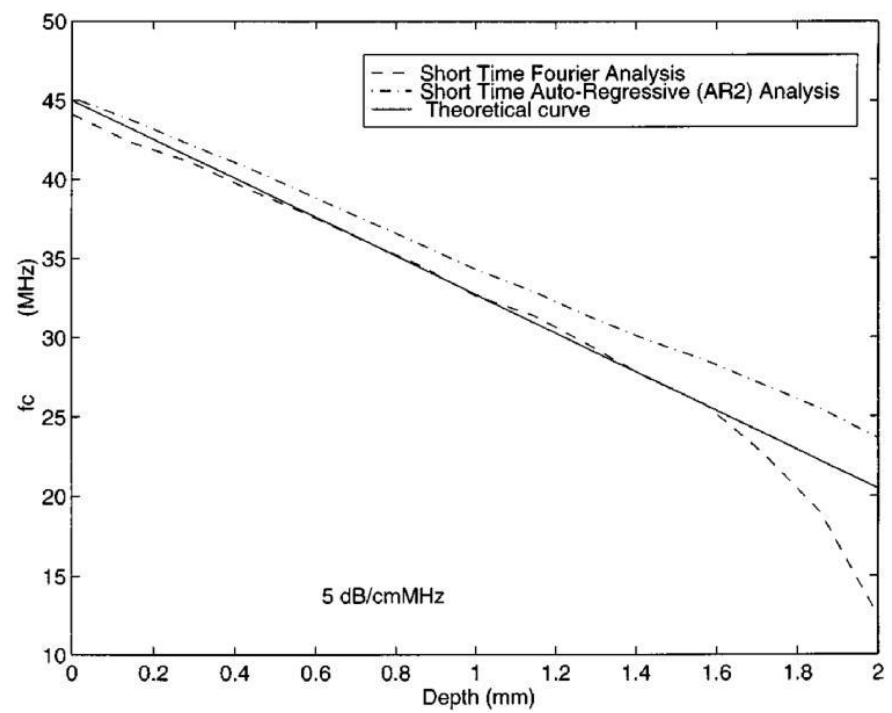

Fig. 5. Evolution of the center frequency, $f_{C}$ (centroid frequency) evaluated by the short-time Fourier approach (dashed line) and by the short-time auto-regressive (AR2) approach (dashdot line) versus depth. The theoretical curve calculated by (5) is illustrated by the solid line. The proposed curves are averaged with 256 radio-frequency lines. The simulation conditions are: $f_{0}=45 \mathrm{MHz}, s=7: 46 \mathrm{MHz}=5$ $\mathrm{dB} / \mathrm{cmMHz}$, and $f_{S}=400 \mathrm{MHz}$.

Owing to the recursive nature of the algorithm, the coe cients become constant after the convergence region. Just like the $a_{i ; g}$ coefficients, the AR parameters (9) and the frequency estimates have a transient region

\section{Spectral Estimation}

From known AR parameters, a standard approach con-sists in computing rst the whole spectrum then estimat-ing a particular frequency (the centroid frequency or the maximum energy frequency).

Accordingly, the power spec-tral density $S_{X X}(f ; n)$ of a nonstationary AR process is given by:

$$
S_{x x}(f, n)=\frac{\sigma_{u}^{2}(n)}{\left|1+\sum_{i=1}^{p} a_{i}(n) e^{-j 2 \pi f i}\right|^{2}}
$$

where $f$ is the normalized frequency $0 f 0: 5$, and ${ }_{u}{ }^{2}(n)$ is the noise power at time $n$. Extension to the time-varying AR model, this expression becomes:

$S_{x x}(f, n)=\frac{\sigma_{u}^{2}(n)}{\left|1+\sum_{i=1}^{p} \sum_{g=0}^{m} a_{i, g} F_{g}(n) e^{-j 2 \pi f i}\right|^{2}}$.

An interesting alternative is to evaluate frequencies. In this case, only one frequency has to be esti-mated instead of the whole spectrum. This implies an im-portant reduction of the computing time. In other words, attenuation information that appears in the time-varying spectrum can be derived from many particular frequencies such as the centroid frequency, the maximum energy fre-quency (the frequency that corresponds to the maximum energy of the spectrum), or the resonating frequency. Here, we develop the mathematical relationships involved in the second-order AR model to evaluate the spectral shift, and particularly we
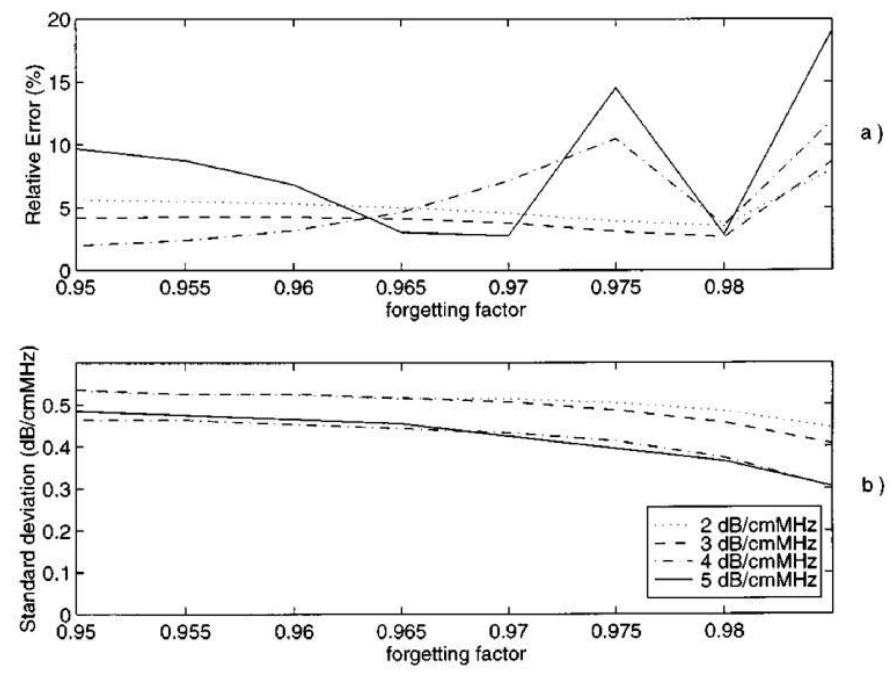

Fig. 6. Evolution of the relative error (a) and the standard devia-tion (b) of the attenuation coefficient estimate versus the forgetting factor used in the adaptive approach. The simulation conditions are: $f_{0}=45 \mathrm{MHz}$, $s=7: 46 \mathrm{MHz},=2,3,4,5 \mathrm{~dB} / \mathrm{cmMHz}$, and $f_{S}=400 \mathrm{MHz}$. The illustrated results are averaged with 256 radio-frequency lines. $=2$ (dotted line), = 3 (dashed line), = 4 (dashdot line), and = 5 (solid line).

will focus on the maximum energy fre-quency [3]: it is obtained by di erentiating (31) with re-spect to $f$ and setting it to zero:

$f_{\max }(n)=\frac{f_{s}}{2 \pi} \cos ^{-1}\left(\frac{-a_{1}(n)}{4}\left(1+\frac{1}{a_{2}(n)}\right)\right)$

and the resonating frequency [16]: it corresponds to the phase of the complex poles de ned in the transfer function of ultrasonic echoes:

$f_{\text {res }}(n)=\frac{f_{s}}{2 \pi} \tan ^{-1}\left(\sqrt{\frac{4 a_{2}(n)}{a_{1}^{2}(n)}-1}\right)$.

In order to have theoretical references and an idea of the initial values of the AR parameters in the estimation process, we propose the introduction of an approximated theoretical value of AR parameters. From (33) and (34) with two unknowns $a_{1}$ and $a_{2}$, by assuming that the resonating frequency is equal to the maximum energy frequency, we can give a theoretical approximated expression of the two AR parameters:

$$
\begin{aligned}
& a_{1}(n) \approx-2 \cos \left(2 \pi \frac{f_{(n)}}{f_{s}}\right) \\
& a_{2}(n) \approx 1
\end{aligned}
$$

where the variable $f_{(n)}$ may be $f_{\max }$ or $f_{\text {res }}$.

The numerical evaluation of the normalized di erence between (34) and (33) (i.e., between $f_{\text {res }}$ and $f_{\text {max }}$ ) is small [16] for the range of typical values of $a_{1}$ and $a_{2}$ (a typical range for $a_{1}$ is $-1.90<a 1<-1.52$ and for $a_{2}$ is $0.9<a_{2}<1$, the corresponding bandwidth is $20 \mathrm{MHz}<f$ $<45 \mathrm{MHz}$ ). This implies that the resonating frequency of a second-order AR system can be approxi-mately represented by the frequency of the maximum en-ergy. 

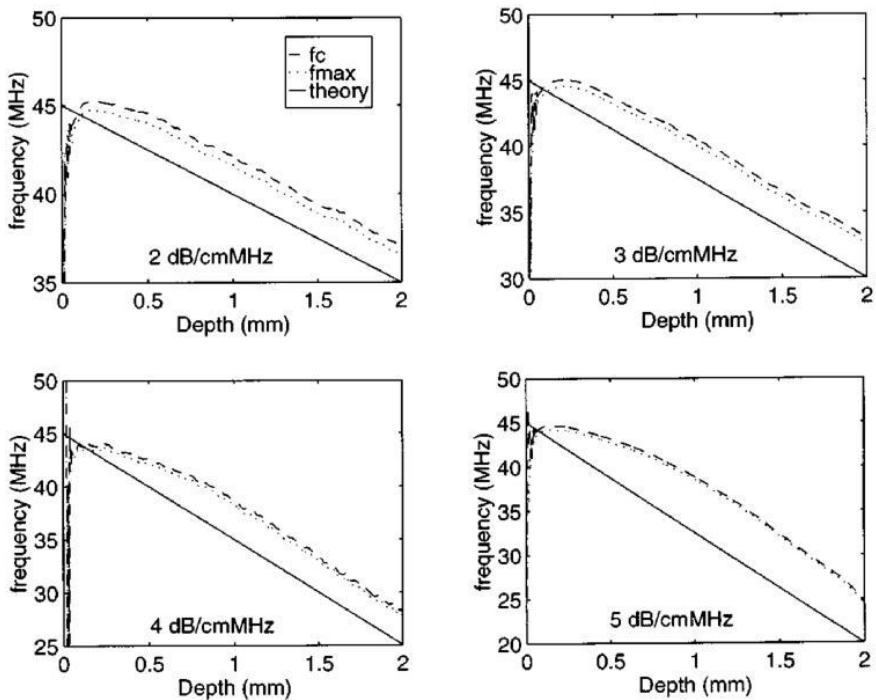

Fig. 7. Evolution of $f_{\max }$ (dotted line) and $f_{C}$ (dashed line) versus depth obtained by the adaptive approach. The theoretical curve calculated by (5) is illustrated by the solid line. Those proposed curves are averaged with 256 radio-frequency lines. The simulation conditions are: $f_{0}=45 \mathrm{MHz}, s=7: 46 \mathrm{MHz},=2,3,4,5 \mathrm{~dB} / \mathrm{cmMHz}$, and $f_{S}=$ $400 \mathrm{MHz}$.

Consequently, as their values are very similar, we only focus on the maximum energy frequency.

By using (6), (35) becomes :

$$
a_{1}(d) \approx-2 \cos \left(2 \pi \frac{f_{0}}{f_{s}}-\frac{8 \pi \sigma_{s}^{2}}{4.34 f_{s}} \beta_{d}\right)
$$

where $d$ is the depth, is the attenuation coefficient, $s$ is the spectral variance.

Note that the initial values of the AR parameters depend on the transducer center frequency $f_{0}$ and the sam-pling frequency $f_{s}$. With (35) and (36) we determine the initial values of $a_{1}$ and $a_{2}$ at time $n=0$ :

$$
\begin{aligned}
& a_{1}(0) \approx-2 \cos \left(2 \pi f_{0} / f_{s}\right)=-1.52 \\
& a_{2}(0)=1 \\
& \theta_{0}=\left[\begin{array}{ll}
a_{1}(0) & a_{2}(0)
\end{array}\right]^{T}=\left[\begin{array}{ll}
-1.52 & 1
\end{array}\right]^{T}
\end{aligned}
$$

with $f_{s}=400 \mathrm{MHz}$ and $f_{0}=45 \mathrm{MHz}$.

Here, we emphasize, in a theoretical manner, the fact that an accurate estimation of , via the computation of the linear regression, cannot be carried out without an accurate estimation of the AR parameters. This means that estimation of the AR parameters must be performed with great care. From (6), it is obvious that variance of is proportional to variance of $f_{\max }$ :

$$
\sigma_{\beta}^{2}=\sigma_{f_{\max }}^{2} K
$$

According to Appendix B, Taylor expansion of the variance of $f_{\max }$ is:

$$
\sigma_{f_{\max }}^{2}=\frac{f_{s}^{2} \hat{a}_{1}\left(-\hat{a}_{2}^{2}\left(\hat{a}_{2}+1\right)^{2} \sigma_{a_{1}}^{2}+\hat{a}_{2}\left(\hat{a}_{2}+1\right) \sigma_{a_{1}, a_{2}}-\sigma_{a_{2}}^{2}\right)}{4 \pi^{2} \hat{a}_{2}^{2}\left(\hat{a}_{1} \hat{a}_{2}+4 \hat{a}_{2}+\hat{a}_{1}\right)\left(\hat{a}_{1} \hat{a}_{2}-4 \hat{a}_{2}+\hat{a}_{1}\right)(42)}
$$

where $\hat{a}_{1}, \hat{a}_{2}$ are two AR estimated parameters with variances $\sigma_{a}{ }_{1}, \sigma_{a} 2$ and covariance $a 1 ; a 2$.

Determination of statistical properties of time-varying frequency estimates is obtained in the same way as in the adaptive case. However, we have to replace
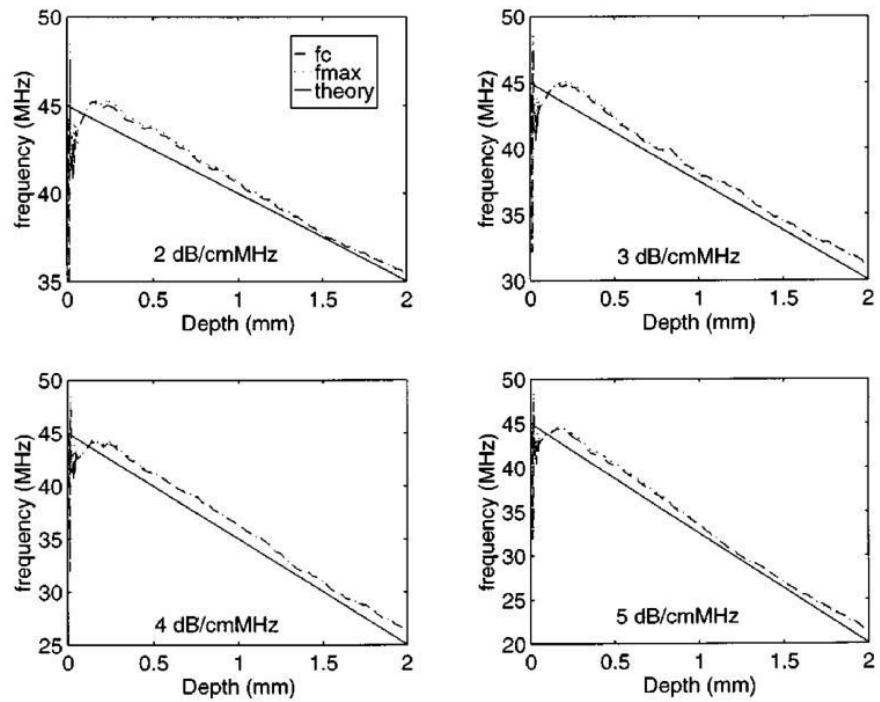

Fig. 8. Evolution of $f_{\max }$ (dotted line) and $f_{C}$ (dashed line) versus depth obtained by the time-varying approach versus depth. The the-oretical curve calculated by (5) is illustrated by the solid line. Those proposed curves are averaged with 256 radio-frequency lines. The simulation conditions are: $f_{0}=45 \mathrm{MHz}, s=7: 46 \mathrm{MHz},=2,3,4,5 \mathrm{~dB} / \mathrm{cmMHz}$, and $f_{S}=400 \mathrm{MHz}$.

variance, co-variance, and bias of each parameter by statistical proper-ties of each basis coefficient.

As expected, (39) shows that the precision (related to variance) of attenuation coefficient estimate is greatly de-pendent on the precision of the AR parameter estimates.

\section{Interpretation and Discussion}

In order to characterize estimates, we focus our attention on the bias and the variance of estimation. To take into account the stochastic variation of the signal, these two statistical characteristics are examined as a function of depth. More precisely, the bias is calculated as the expectation of the di erence between the average estimated value and the theoretical value at each depth and the computation of variance is based on the same principle.

\section{A. Statistical Analysis of Frequency Estimates}

Fig. 7 illustrates the evolution of $f_{\max }(33)$ and $f_{C}$ [5] versus depth obtained by the adaptive algorithm. These curves are averaged with 256 radio-frequency (RF). At the beginning of the frequency estimation, a transient region appears that is due to the recursive nature of the algo-rithm. However, the curves of frequency estimates are al-most parallel to those of theoretical frequencies.

Although we have increased the number of unknowns, the time-varying method leads to improvement of parameters estimation accuracy for a time-varying signal. Indeed, Fig. 8, which presents the evolution of $f_{\max }$ and $f_{C}$, well illustrates that the curves of frequency estimates are almost parallel to the theoretical frequencies.

Fig. 9 shows the bias and the mean square error of $f_{\max }$ evaluated by the adaptive method.

The analysis of results shown in Figs. 7, 8, and 9 lead to the following observations: 

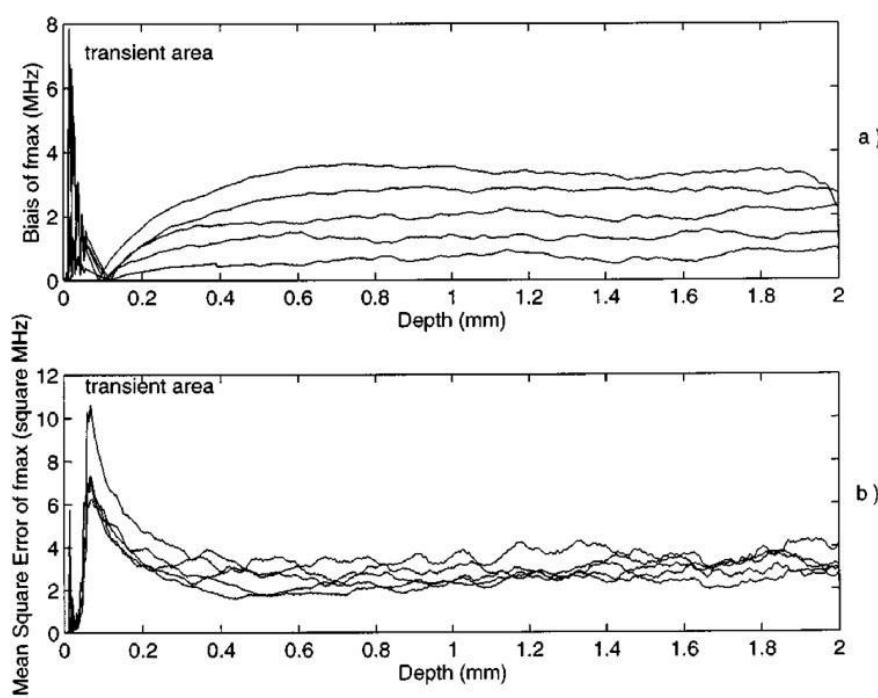

Fig. 9. Evolution of the bias (a) and the mean square error (b) of $f_{\max }$ versus depth by using the adaptive approach. The simulation conditions are: $f_{0}=45 \mathrm{MHz}, s=7: 46 \mathrm{MHz},=1,2,3,4,5 \mathrm{~dB} / \mathrm{cmMHz}$, and $f_{S}$ $=400 \mathrm{MHz}$. The gap between the theoretical curve and the estimate increases with the increasing value of but not with the depth. Variance seems independent of the attenuation value and the depth.

- Except the transient region, the bias and the variance of the frequency estimates are roughly independent of the explored medium depth. This trend also has been veri ed with the parameter estimates. This phe-nomenon can be simply explained thanks to the homogeneity of the medium and the lack of di raction ef-fects in the simulations. Consequently, this result con- rms that the estimation of can be obtained via the computation of the slope of $f_{\max }$ along the explored medium.

- The systematic error on the frequency estimation (bias) is dependent on attenuation in the adaptive ap-proach, whereas in the time-varying approach it seems relatively independent on attenuation. This behavior is explained by the fact that the AR parameters eval-uated by the adaptive approach are much more biased than the ones estimated by the time-varying approach. The attenuation-dependence of frequency bias does not disturb attenuation estimation because its slope constitutes the relevant information (because the fre-quency bias is constant versus depth).

- The variance of the frequency estimator, evaluated by the adaptive algorithm, is relatively independent on the attenuation. The same trend has been observed for the time-varying algorithm. Indeed, the variance of parameters are independent of depth. Accordingly, we can foresee that variance of is constant regardless of the di erent attenuation values.

Estimation obtained either by using the maximum en-ergy frequency or the centroid frequency provides close results. This is due to the smoothness of the AR PSD. However, the
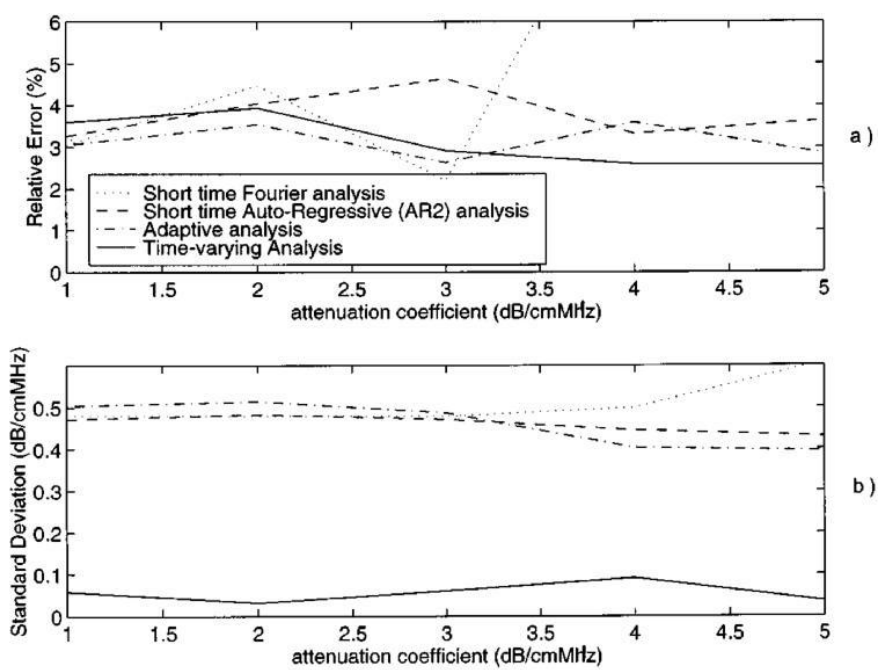

Fig. 10. Evolution of the relative error (a) and the standard de-viation (b) of the attenuation coefficient estimate versus the the-oretical attenuation coefficient values and for di erent approaches.

computation of $f_{\max }$ is faster than that of $f_{C}$, which explains our choice of $f_{\max }$.

\section{B. Statistical Analysis of Attenuation}

\section{Coefficient Estimates}

Fig. 10 shows the relative error and standard deviation for short-time Fourier, short-time AR, adaptive $A R$ and time-varying AR methods. The following observations can be made:

- In the case of the short-time Fourier analysis, evaluat-ing the attenuation coefficient by the use of the cen-troid frequency is by far more e cient than by using the maximum energy frequency. Indeed, $f_{C}$ represents an average value of the frequency distribution.

- Because $f_{C}$ and $f_{\max }$ evaluated by the AR approach are similar, the corresponding attenuation coefficients are very close (Table I).

- Concerning the attenuation estimate, results obtained using $f_{C}$ with the short-time Fourier analysis show a high variance compared to those obtained by the para-metric approaches. In addition, the $\mathrm{RE}$ reaches and even exceeds $10 \%$ for $=4 \mathrm{~dB} / \mathrm{cmMHz}$ whereas the RE given by the parametric approaches is below 5\%. Fig. 10(b) shows that standard deviation using the adaptive approach is lower than short-time analysis for high attenuations.

- Time-varying method is better than adaptive and batch methods in terms of SD whereas the $R E$ is nearly the same for all methods.

Hence, by using a time-varying method, we can improve the standard deviation of short-time Fourier analysis by a factor 5 . This means that the averaging operation can be 
TABLE I

The RE and the SD of the Attenuation Coefficient Evaluated via $f_{\max }$ and $f_{C}$ for Different Attenuations ${ }^{1}$.

\begin{tabular}{cccccc}
\hline $\begin{array}{c}\text { Attenuation coe cient } \\
(\mathrm{dB} / \mathrm{cmMHz})\end{array}$ & 1 & 2 & 3 & 4 & 5 \\
\hline Relative Error of $: f_{\max }$ & 3.055 & 3.546 & 2.633 & 3.592 & 2.847 \\
$\begin{array}{c}(\%) \\
\text { Relative Error of }: f_{C}\end{array}$ & 2.012 & 1.951 & 1.972 & 3.33 & 2.368 \\
$\begin{array}{c}(\%) \\
\text { Standard Deviation of }: f_{\max } \\
\quad(\mathrm{dB} / \mathrm{cmMHz})\end{array}$ & 0.474 & 0.485 & 0.456 & 0.373 & 0.365 \\
$\begin{array}{c}\text { Standard Deviation of }: f_{C} \\
(\mathrm{~dB} / \mathrm{cmMHz})\end{array}$ & 0.496 & 0.493 & 0.489 & 0.392 & 0.375 \\
\hline
\end{tabular}

\footnotetext{
The results are very close; however, we note some di erences: RE of evaluated via $f_{C}$ is better than the one obtained via $f_{\max }$, whereas the SD of evaluated via $f_{\text {max }}$ is better than the one obtained via $f_{C}$.
}

performed with less uncorrelated RF signals than commonly used.

The a posteriori knowledge of RE means that it becomes possible to easily correct this error on the attenuation es-timation.

\section{Conclusion}

We have proposed two new approaches for ultrasound attenuation estimation: adaptive AR2 and time-varying AR2. Both the time-varying approach, whose basis is a two-dimensional power time function, and the adaptive approach provide bias and variance on spectral estimation that are independent of the explored tissue depth. These two approaches, which are radically di erent in their prin-ciple, give a bias on the frequency estimate that is depen-dent on the attenuation coefficient value and a variance of the frequency estimate that is only weakly a ected by attenuation.

Concerning the attenuation coefficient estimate, in terms of accuracy, the performances of the time-varying method are far superior to those of short-time Fourier and short-time AR analyses. Furthermore, the superiority of the time-varying approach compared to the adaptive method indicates that it is very well adapted to nonstationary signals encountered both in weakly and highly attenuating media.

The joint use of high frequency and time-varying analysis is a promising way to evaluate tissue attenuation that has to be con rmed by both in vitro and in vivo measurements. Potential applications are in dermatology and ophthalmology where tissue attenuation can be related to a pathological state.

To better analyze the attenuation, it may be interesting to consider an adaptive algorithm using higher order statistics already introduced in signal processing [17]

\section{Acknowledgements}

We gratefully acknowledge Pr. Marc Lethiecq and $\mathrm{Dr}$ Christelle Guittet of University of Tours for the help with the manuscript. We are also indebted to anonymous reviewers for their helpful comments.

\section{Appendix A}

The dierent basis functions proposed are:

- Legendre functions:

$$
\begin{aligned}
F_{g}(n)=\frac{2 g-1}{g}\left(2 \frac{n}{N}-1\right) & F_{g-1}(n-1) \\
& -\frac{g-1}{g} F_{g-2}(n) .
\end{aligned}
$$

- Power time functions:

$$
F_{g}(n)=\frac{1}{g !}\left(\frac{n}{N}\right)^{g} \text {. }
$$

- Trigonometric functions:

$$
F_{g}(n)=\cos (2 \pi f g n) \text { if } g \text { is even }
$$

$$
F_{g}(n)=\sin (2 \pi f g n) \text { if } g \text { is odd }
$$

where $F_{0}(n)=1$.

\section{Appendix B}

The Taylor expansion of the variance of $f \max [18]$ with the help of (33) is given by:

$$
\begin{array}{r}
\sigma_{f_{\max }}^{2} \approx\left(\frac{\partial f_{\max }}{\partial \hat{a}_{1}}\right)^{2} \sigma_{a_{1}}^{2}+2\left(\frac{\partial f_{\max }}{\partial \hat{a}_{1}}\right)\left(\frac{\partial f_{\max }}{\partial \hat{a}_{2}}\right) \sigma_{a_{1}, a_{2}} \\
+\left(\frac{\partial f_{\max }}{\partial \hat{a}_{2}}\right)^{2} \sigma_{a_{2}}^{2}
\end{array}
$$

with

$$
\begin{aligned}
& \left(\frac{\partial f_{\max }}{\partial \hat{a}_{1}}\right)^{2}=\frac{f_{s}^{2} \hat{a}_{1}\left(\hat{a}_{2}+1\right)^{2}}{-4 \pi^{2}\left(\hat{a}_{1} \hat{a}_{2}+4 \hat{a}_{2}+\hat{a}_{1}\right)\left(\hat{a}_{1} \hat{a}_{2}-4 \hat{a}_{2}+\hat{a}_{1}\right)} \\
& \left(\frac{\partial f_{\max }}{\partial \hat{a}_{2}}\right)^{2}=\frac{f_{s}^{2} \hat{a}_{1}}{-4 \pi^{2} \hat{a}_{2}^{2}\left(\hat{a}_{1} \hat{a}_{2}+4 \hat{a}_{2}+\hat{a}_{1}\right)\left(\hat{a}_{1} \hat{a}_{2}-4 \hat{a}_{2}+\hat{a}_{1}\right)}
\end{aligned}
$$

$$
\begin{aligned}
& \left(\frac{\partial f_{\max }}{\partial \hat{a}_{2}}\right)\left(\frac{\partial f_{\max }}{\partial \hat{a}_{1}}\right)= \\
& \quad \frac{f_{s}^{2} \hat{a}_{1}\left(\hat{a}_{2}+1\right)}{4 \pi^{2} \hat{a}_{2}\left(\hat{a}_{1} \hat{a}_{2}+4 \hat{a}_{2}+\hat{a}_{1}\right)\left(\hat{a}_{1} \hat{a}_{2}-4 \hat{a}_{2}+\hat{a}_{1}\right)} .
\end{aligned}
$$




\section{References}

[1] M. Lethiecq, G. Feuillard, M. Berson, and F. Patat, "Principles and applications of high frequency medical imaging," in Advances in Acoustic Microscopy, vol. 2, A. Briggs, and W. Arnold, Eds. NewYork: Plenum, pp. 39-102, 1996.

[2] F. K. Forster, J. E. Olerud, M. A. Riederer-Henderson, and A. W. Holmes, "Ultrasonic assessment of skin and surgical wounds utilizing backscatter acoustic techniques to estimate attenuation," Ultrason. Med. Biol., vol. 16, pp. 43-53, 1990.

[3] R. Kuc and H. Li, "Reduced-order auto-regressive modeling forcenter frequency estimation," Ultrason. Imaging, pp. 244-251, 1985.

[4] G. Berger, P. Laugier, M. Fink, and J. Perrin, "Optimal precision in ultrasound attenuation estimation and application to the detection of Duchenne muscular dystrophy carriers," Ultrason. Imaging, vol. 9, pp. 1-17, 1987.

[5] T. Baldeweck, P. Laugier, A. Herment, and G. Berger, "Application of auto-regressive spectral analysis for ultrasound attenuation estimation: Interest in highly attenuation medium," IEEE Trans. Ultrason., Ferroelect., Freq. Contr., vol. 42, no. 1, pp. 99-110, 1995.

[6] B. J. Oosterveld, J. M. Thijssen, and W. A. Verhoef, "Texture of Bmode echograms: 3-D simulations and experiments of the e ects of di raction and scattering density," Ultrason. Imaging, vol. 7, pp. 142160, 1985

[7] M. Fink, F. Hottier, and J. F. Cardoso, "Ultrasonic signal processing for in vivo attenuation measurement: Short time Fourier analysis," Ultrason. Imaging, vol. 5, pp. 117-135, 1983.
[8] L. Ferrari, J. P. Jones, V. Gonzalez, and M. Behrence, In vivo measurement of attenuation based on the Gaussian pulse propagation," Ultrason. Imaging, vol. 5, pp. 95-116, 1983.

9] S. M. Kay and S. L. Marple, "Spectrum analysis-A modern perspective," Proc. IEEE, vol. 69, no. 11, pp. 1380-1419, 1981.

[10] Y. Grenier, "Time-dependent ARMA modeling of non-stationary signal," IEEE Trans. Acoust., Speech, Signal Processing, vol. 31, no. 4, pp. 899\{911, 1983.

11] L. A. Liporace, "Linear estimation of non-stationary signal," J. Acoust. Soc. Amer., vol. 58, no. 6, pp. 1288-1295, 1975.

[12] M. Hall, A. V. Oppenheim and A. Willsky, "Time-varying para metric modeling of speech," Signal Processing, vol. 5, no. 3, pp. 267-285, 1983.

[13] L. Ljung, Recursive Identi cation: Theory for the Users. Engle-wood Cli s, NJ: Prentice-Hall, 1987.

[14] L. Ljung and S. Gunnarsson, VAdaptation and tracking in sys-tem identi cation. A survey," Automatica, vol. 26, no. 1, pp. 7-21, 1990.

[15] K. A. Wear, R. F. Wagner, and B. S. Garra, "High resolu-tion ultrasonic backscatter coe cient estimation based on autoregressive spectral estimation using Burg's algorithm," IEEE Trans. Med. Imag., vol. 13, no. 3, pp. 500-507, 1994.

16] T. Wang, J. Saniie, and X. Jin, VAnalysis of low-order autoregressive models for ultrasonic grain signal characterization," IEEE Trans. Ultrason., Ferroelect., Freq. Contr., vol. 38, no. 2, pp. 116124, 1991.

[17] D. Aboutajdine, A. Adib, and A. Meziane, IFast adaptive algo-rithms for AR parameters estimation using higher order statis-tics," IEEE Trans. Signal Processing, vol. 44, no. 10, pp. 2498-2507, 1996.

[18] Papoulis, Probability, Random Variables, and Stochastic Processes, 3nd ed. New York: McGraw-Hill, p. 156, 1991. 